\title{
Clinical Study \\ Beneficial Effects of Testosterone Therapy on Functional Capacity, Cardiovascular Parameters, and Quality of Life in Patients with Congestive Heart Failure
}

\author{
Ahmad Mirdamadi, ${ }^{1}$ Mohammad Garakyaraghi, ${ }^{2}$ Ali Pourmoghaddas, ${ }^{2}$ \\ Alireza Bahmani, ${ }^{1}$ Hamideh Mahmoudi, ${ }^{3}$ and Mojgan Gharipour ${ }^{4}$ \\ ${ }^{1}$ Islamic Azad University, Najafabad Branch, Isfahan, Iran \\ ${ }^{2}$ Heart Failure Research Center, Isfahan Cardiovascular Research Institute, Isfahan University of Medicine Science, Isfahan, Iran \\ ${ }^{3}$ Cardiac Rehabilitation Research Center, Isfahan Cardiovascular Research Institute, Isfahan University of Medicine Science, \\ Isfahan, Iran \\ ${ }^{4}$ Isfahan Cardiovascular Research Center, Isfahan Cardiovascular Research Institute, Isfahan University of Medicine Science, \\ Isfahan, Iran
}

Correspondence should be addressed to Mohammad Garakyaraghi; garagyaraghi@med.mui.ac.ir

Received 14 February 2014; Accepted 5 May 2014; Published 6 July 2014

Academic Editor: Kimimasa Tobita

Copyright (C) 2014 Ahmad Mirdamadi et al. This is an open access article distributed under the Creative Commons Attribution License, which permits unrestricted use, distribution, and reproduction in any medium, provided the original work is properly cited.

\begin{abstract}
Background. According to the present evidences suggesting association between low testosterone level and prediction of reduced exercise capacity as well as poor clinical outcome in patients with heart failure, we sought to determine if testosterone therapy improves clinical and cardiovascular conditions as well as quality of life status in patients with stable chronic heart failure. Methods. A total of 50 male patients who suffered from congestive heart failure were recruited in a double-blind, placebo-controlled trial and randomized to receive an intramuscular (gluteal) long-acting androgen injection ( $1 \mathrm{~mL}$ of testosterone enanthate $250 \mathrm{mg} / \mathrm{mL}$ ) once every four weeks for 12 weeks or receive intramuscular injections of saline $(1 \mathrm{~mL} \mathrm{of} 0.9 \% \mathrm{wt} / \mathrm{vol} \mathrm{NaCl})$ with the same protocol. Results. The changes in body weight, hemodynamic parameters, and left ventricular dimensional echocardiographic indices were all comparable between the two groups. Regarding changes in diastolic functional state and using Tei index, this parameter was significantly improved. Unlike the group received placebo, those who received testosterone had a significant increasing trend in 6-walk mean distance (6MWD) parameter within the study period $(P=0.019)$. The discrepancy in the trends of changes in $6 \mathrm{MWD}$ between study groups remained significant after adjusting baseline variables (mean square $=243.262, F$ index $=4.402$, and $P=0.045$ ). Conclusion. Our study strengthens insights into the beneficial role of testosterone in improvement of functional capacity and quality of life in heart failure patients.
\end{abstract}

\section{Introduction}

Heart failure is a serious cardiovascular condition leading to life-threatening events, poor prognosis, and degradation of quality of life. In some studies, considerable morbidity and excessive mortality with a rate up to $30 \%$ has been annually exhibited [1]. In this regard, treatment and management of affected patients particularly those with progressive left ventricular dysfunction are a main problem and thus the use of single-drug treatment protocols or preventive approaches is mostly unpromising [2]. Recently, a noticeable evolution of therapeutic concepts has taken place with a variety of cardiac and hormonal drugs with the aim of improving patient's survival, preventing sudden death, and improving quality of life $[3,4]$. In a significant proportion of heart failure patients, testosterone deficiency as an anabolic hormonal defect has been proven and identified even in both genders [5]. This metabolic and endocrinological abnormality is frequently associated with impaired exercise tolerance and reduced cardiac function [6]. For this reason, combination 
therapy with booster cardiovascular drugs and testosterone replacement therapy might be very beneficial in heart failure patients. The physiological pathways involved in these therapeutic processes have been recently examined. First, elevated level of testosterone following replacement therapy is major indicator for increase of peak $\mathrm{VO}_{2}$ in affected men with heart failure explaining improvement of exercise tolerance in these patients $[7,8]$. Furthermore, testosterone replacement therapy can reduce circulating levels of inflammatory mediators including tumor necrosis factor $\alpha$ (TNF- $\alpha$ ) and interleukin (IL) $-1 \beta$, as well as total cholesterol in patients with established simultaneous coronary artery disease and testosterone deficiency $[9,10]$.

According to the present evidences suggesting association between low testosterone level and prediction of reduced exercise capacity as well as poor clinical outcome in patients with heart failure, we sought to determine if testosterone therapy improves clinical and cardiovascular conditions as well as quality of life status in patients with stable chronic heart failure.

\section{Methods}

This study was designed as a double-blind, placebo-controlled trial, with 50 male patients who suffered from congestive heart failure that is defined as an ejection fraction less than $40 \%$ on echocardiogram or clinical heart failure according to the Framingham's criteria for diagnosis of heart failure $[11,12]$ and recruited from 2011 to 2013 . Inclusion criteria were age range 50 to 70 years and suffering congestive heart faire. Exclusion criteria were history or symptoms of adrenal or gonad disorders, recent history of unstable angina, myocardial infarction, uncontrolled hypertension, severe liver diseases, kidney disease, erythrocytosis (serum hematocrit > $50 \%$ ), prostatic disorders, or international prostate symptom score (IPSS) more than 20. This research was approved by the Research and Ethics Committees at Isfahan University of Medical Sciences. Structured baseline interviews were carried out by a research assistant within hospitalization including questions about social demographic characteristics and as well as cardiovascular risk factors. Conventional cardiac risk factors were identified, including current smoking history (patients who regularly smoke tobacco product/products one or more times per day or have smoked in the 30 days prior to admission) [13], hypercholesterolemia (total cholesterol $\geq 5.0 \mathrm{mmoL} / \mathrm{l}$, HDL-cholesterol $<1.0 \mathrm{mmoL} / \mathrm{l}$ in men or $<1.1 \mathrm{mmoL} / 1$ in women, and triglycerides $\geq 2.0 \mathrm{mmoL} / \mathrm{l}$ ) [14], family history of CAD (first-degree relatives before the age of 55 in men and 65 years in women) [15], hypertension (systolic blood pressure $\geq 140 \mathrm{mmHg}$ and/or diastolic $\geq 90 \mathrm{mmHg}$ and/or on antihypertensive treatment) [16], and diabetes mellitus (symptoms of diabetes plus at least one of the following: plasma glucose concentration $\geq 11.1 \mathrm{mmoL} / \mathrm{l}$, fasting plasma glucose $\geq 7.0 \mathrm{mmoL} / \mathrm{l}$, and 2-hpp $\geq 11.1 \mathrm{mmoL} / \mathrm{l}$ ) [17]. Anthropometric parameters were measured using standard protocols. Functional limitation was assessed clinically according to the New York Heart Association (NYHA) classification and functional capacity was assessed by means of six-minute walk test (6MWT), that is, a symptom-limited exercise test involving a progressive increase in walking speed to maximal exercise tolerance [18]. Morphologic and functional changes in cardiac structure and function were assessed using an ultrasound device and dedicated $3.5 \mathrm{MHz}$ phased-array ultrasonic transducer according to the American Society of Echocardiography guidelines for measuring left ventricular end systolic and end diastolic dimensions and volumes as well as systolic and diastolic functions, left ventricular filling pressure, and Tei index [19]. For assessment of quality of life state, the Short Form Health Survey (SF-36) questionnaire was used that the total score was calculated based on eight physical and psychological subscales and with a higher score indicating a better QOL [20]. Depressive symptoms were assessed using the Persian version of the Beck Depression Inventory (BDI) that was validated among Iranian cardiovascular population with the Cronbach's alpha of 0.92 for reliability and intraclass correlation coefficient of 0.72 for validity assessment [21]. BDI is a self-reported questionnaire, which is used for routine assessment of the severity of depression in psychiatric patients and for the screening for depressive symptoms in the general population. BDI consists of 21 items that cover emotional, behavioral, and somatic symptoms. The total score ranges from 0 to 63 points (scored $0-3$ for each item) [22]. In this study, the $6 \mathrm{~min}$ walk test was conducted according to a standardized protocol and the 6 min walk distance (6MWD) was measured. Upper extremity strength was also assessed according to grip strength using the dynamometer. Grip strength involved two trials on each hand. The mean value of the two trials was obtained. For this analysis, the maximum strength in either hand was used for upper extremity strength [23]. Patients were randomized to receive an intramuscular (gluteal) long-acting androgen injection $(1 \mathrm{~mL}$ of testosterone enanthate $250 \mathrm{mg} / \mathrm{mL}$ ) once every 4 weeks for 12 weeks or receive intramuscular injections of saline ( $1 \mathrm{~mL}$ of $0.9 \% \mathrm{wt} / \mathrm{vol} \mathrm{NaCl}$ ) with the same protocol. Hence, all patients received 3 injections over the 12 -week period. Measurements to the study including assessment of echocardiographic parameters, quality of life and depression states, and muscle strength were performed before intervention and also as endpoint after 12 weeks. Also, the changes in body weight, functional capacity, and hemodynamic indicators were assessed at baseline and also at the end of each month in both groups and compared. Results were reported as mean \pm standard deviation (SD) for the quantitative variables and percentages for the categorical variables. The groups were compared using the Student's $t$-test or Mann-Whitney $U$ test for the continuous variables and the chi-square test (or Fisher's exact test if required) for the categorical variables. The changes in the study parameters within study period were assessed and compared using the repeated measure ANOVA test. In this analysis, the effect size (indicated by $F$-index) indicated within- and between-subjects variability. The interpretation of the $F$-index is considered large (or significant) if it is higher than 0.40 , medium if it ranged between 0.25 and 0.40 , and small if it is lower than 0.25 . For determining differences in the trends of study parameters between the two groups, the results of the repeated measure ANOVA test were adjusted for baseline variables. In this regard, $P$ values of 0.05 or less for each variable indicated discrepancy 
TABLE 1: Comparison of baseline information between intervention and placebo groups.

\begin{tabular}{lccc}
\hline Characteristics & $\begin{array}{c}\text { Testosterone } \\
\text { group } \\
(n=25)\end{array}$ & $\begin{array}{c}\text { Placebo } \\
\text { group } \\
(n=25)\end{array}$ & $P$ value \\
\hline Age, year & $60.83 \pm 8.31$ & $60.24 \pm 11.89$ & 0.841 \\
Body mass index, kg/m ${ }^{2}$ & $26.78 \pm 2.91$ & $26.11 \pm 3.97$ & 0.505 \\
Diabetes mellitus & $7(28)$ & $6(24)$ & 0.747 \\
Hypertension & $7(28)$ & $3(12)$ & 0.289 \\
Hyperlipidemia & $11(44)$ & $6(24)$ & 0.136 \\
Cigarette smoking & $2(8)$ & $1(4)$ & 0.998 \\
NYHA classification & & & 0.272 \\
$\quad$ II & $20(80)$ & $13(52)$ & \\
III & $5(20)$ & $10(40)$ & \\
IV & $0(0)$ & $2(8)$ & \\
\hline
\end{tabular}

in the time trend of the variable between the two groups. The difference in response to treatment protocols across the groups was assessed using a multivariable logistic regression model considering baseline data as probable independent confounders. The beta coefficient in this model was used to compare the strength of a predictor within the model to predict the dependant variable or outcome so that the higher beta indicates more power of the variable to predict outcome. Because beta coefficient is potentially affected by the standard error of the variable, the standardized beta adjusted for this error is considered. $P$ values of 0.05 or less were considered statistically significant. All the statistical analyses were performed using SPSS version 19.0 (SPSS Inc., Chicago, IL, USA) and SAS version 9.1 for Windows (SAS Institute Inc., Cary, NC, USA).

\section{Results}

Comparing baseline variables and clinical parameters across the two groups who received testosterone or placebo (Table 1) did not show any significant difference, except for 6MWD that was higher in the testosterone group. During the 12week study period, no significant differences were revealed in the trend of the changes in hemodynamic parameters including systolic and diastolic blood pressures as well as heart rate between the two groups (Table 2). Also, the changes in body weight were comparable between the groups, while, unlike the group received placebo, those who received testosterone had a significant increasing trend in 6MWD parameter within the study period (6MWD at baseline was $407.44 \pm 100.23 \mathrm{~m}$ and after 12 weeks of followup reached $491.65 \pm 112.88 \mathrm{~m}$ following testosterone therapy, $P=0.019$ ). According to post hoc analysis, the mean 6-walk distance parameter was improved at three time points of 4 weeks, 8 weeks, and 12 weeks after intervention compared with baseline; however no differences were found in this parameter at three postintervention time points. The discrepancy in the trends of changes in 6MWD between study groups remained

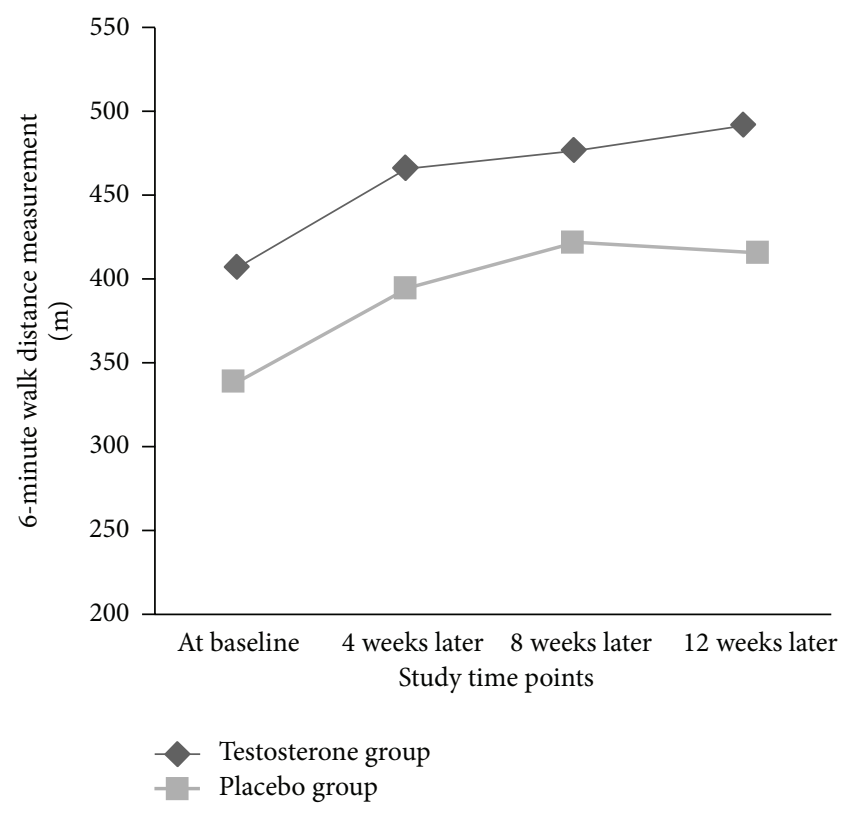

FIGURE 1: Trend of the changes in 6-minute walk distance (6MWD) parameter (in meter) in intervention and placebo groups.

significant after adjusting baseline variables (mean square $=$ 243.262, F-index $=4.402$, and $P=0.045$ ) (Table 3 and Figure 1). The muscle strength was gradually increased in intervention group but not in control; however, this trend was not different across the two groups (Table 2). Among cardiovascular parameters assessed by echocardiography, no differences were observed between the patients who were prescribed testosterone and those who received placebo from baseline to end of the study time (Table 4). Also, regarding changes in diastolic functional state and using Tei index, this parameter was significantly improved. However, quality of life score was significantly improved in former group after adjustment for confounders using the multivariate linear regression modeling (Table 5).

\section{Discussion}

Hormonal metabolic impairment is a main part of fundamental pathophysiological features in heart failure patients. These endocrinological changes lead to serious clinical and prognostic consequences in these patients. On the top of these variations, abnormalities in the serum level of testosterone have been especially more paid attention to. Reducing testosterone level has been identified as the fundament of andropause or male menopause characterized by the slow but steady reduction of the production of the hormones testosterone and dehydroepiandrosterone in middle-aged men. This phenomenon seems to be more progressive in heart failure state leading inappropriate physical, cardiovascular, or psychological consequences. In a recent report, deficiencies in circulating testosterone were reported in $13 \%$ of heart failure patients that the reduced circulating testosterone was associated with lower heart rate variability and depleted 
TABLE 2: Changes in hemodynamic indices, body weight, and function capacity.

\begin{tabular}{|c|c|c|c|c|}
\hline Item & At baseline & 4 weeks later & 8 weeks later & 12 weeks later \\
\hline \multicolumn{5}{|c|}{ Systolic blood pressure (mmHg) } \\
\hline Testosterone group & $119.92 \pm 16.99$ & $121.60 \pm 12.99$ & $122.84 \pm 9.49$ & $125.92 \pm 15.67$ \\
\hline Placebo group & $126.12 \pm 18.87$ & $122.24 \pm 19.17$ & $121.24 \pm 18.38$ & $120.74 \pm 28.45$ \\
\hline$P$ value & 0.232 & 0.891 & 0.700 & 0.434 \\
\hline \multicolumn{5}{|c|}{ Diastolic blood pressure (mmHg) } \\
\hline Testosterone group & $81.17 \pm 18.77$ & $75.80 \pm 9.70$ & $76.44 \pm 7.58$ & $79.00 \pm 9.99$ \\
\hline Placebo group & $79.04 \pm 8.94$ & $73.76 \pm 8.95$ & $72.68 \pm 19.27$ & $76.13 \pm 8.26$ \\
\hline$P$ value & 0.613 & 0.444 & 0.369 & 0.286 \\
\hline \multicolumn{5}{|l|}{ Heart rate $\left(\mathrm{bpm}^{*}\right)$} \\
\hline Testosterone group & $71.21 \pm 8.83$ & $70.48 \pm 9.49$ & $69.92 \pm 6.84$ & $70.88 \pm 8.78$ \\
\hline Placebo group & $69.52 \pm 7.56$ & $71.28 \pm 7.12$ & $68.56 \pm 8.60$ & $71.52 \pm 7.94$ \\
\hline$P$ value & & 0.738 & 0.539 & 0.792 \\
\hline \multicolumn{5}{|l|}{ Body weight (Kg) } \\
\hline Testosterone group & $76.66 \pm 10.42$ & $75.88 \pm 10.35$ & $75.84 \pm 10.26$ & $75.46 \pm 9.96$ \\
\hline Placebo group & $73.12 \pm 12.67$ & $74.00 \pm 12.47$ & $74.28 \pm 11.92$ & $74.52 \pm 11.92$ \\
\hline$P$ value & 0.286 & 0.565 & 0.622 & 0.768 \\
\hline \multicolumn{5}{|l|}{$6 \mathrm{MWD}^{\dagger}(\mathrm{m})$} \\
\hline Testosterone group & $407.44 \pm 100.23$ & $466.58 \pm 93.33$ & $476.67 \pm 96.59$ & $491.65 \pm 112.88$ \\
\hline Placebo group & $338.25 \pm 125.60$ & $394.17 \pm 98.37$ & $421.52 \pm 99.14$ & $416.09 \pm 121.57$ \\
\hline$P$ value & 0.038 & 0.012 & 0.044 & 0.034 \\
\hline \multicolumn{5}{|l|}{ Muscle strength (kgf) } \\
\hline Testosterone group & $37.64 \pm 9.64$ & $39.60 \pm 8.19$ & $41.60 \pm 9.57$ & $42.12 \pm 9.06$ \\
\hline Placebo group & $35.17 \pm 10.54$ & $36.68 \pm 6.87$ & $38.88 \pm 7.73$ & $38.65 \pm 8.17$ \\
\hline$P$ value & 0.411 & 0.178 & 0.278 & 0.170 \\
\hline
\end{tabular}

${ }^{*}$ Beats per minute.

${ }^{\dagger}$ Six-minute walk distance.

Data are presented as mean $\pm \mathrm{SD}$.

TABLE 3: Improvement in 6MWD following testosterone therapy.

\begin{tabular}{lcc}
\hline Item & $F$-index & $P$ value $^{\dagger}$ \\
\hline Testosterone therapy & 4.402 & 0.045 \\
Advanced age & 5.135 & 0.031 \\
Body mass index & 6.035 & 0.020 \\
Diabetes mellitus & 1.786 & 0.192 \\
Hypertension & 0.064 & 0.803 \\
Hyperlipidemia & 0.113 & 0.739 \\
Cigarette smoking & 1.203 & 0.282 \\
NYHA classification & 1.592 & 0.217 \\
\hline
\end{tabular}

Adjusted for baseline variables using the repeated measure ANOVA test.

${ }^{*} F$-index: the interpretation of the $F$-index (indicating the level of variability between subjects) is considered significant if it is higher than 0.40 .

${ }^{\dagger} P$ values of 0.05 or less for each variable indicated discrepancy in the time trend of the variable between the two groups.

baroreflex sensitivity [24]. In this line, it has been observed that low levels of growth hormone and testosterone have been associated with increased mortality and morbidity in patients with heart failure $[25,26]$. Thus, improvement in different cardiovascular indices followed by different aspects of quality of life and psychological status after testosterone therapy can be expectable. In the present study and among study parameters, 6MWD was significantly increased following testosterone prescription. Furthermore, those patients receiving testosterone experienced considerable improvement in quality of life that was not indicated in placebo group. In a similar study by Stout and colleagues, comparable improvements in shuttle walk test, body mass, and hand grip strength from baseline were shown in both testosterone and placebo groups. However, peak oxygen uptake, Beck Depression Inventory, leg strength, and several quality of life components were more improved in former interventional group [27]. Similarly, Iellamo et al. showed that $6 \mathrm{MWD}$, peak oxygen consumption, maximal voluntary contraction, and peak torque increased significantly in testosterone group but remained unchanged in control group [28]. In a recent meta-analysis, a significant increase was shown in exercise capacity following testosterone therapy [29]. Also, it has been suggested that low circulating testosterone independently relates to exercise intolerance that the greater reduction of serum testosterone is accompanied with more severe progression of exercise intolerance [30].

A variety of underlying mechanisms involved in the beneficial effects of testosterone in heart failure condition have been investigated. Some improvement in hemodynamic parameters following injection of testosterone has been demonstrated to be mediated by reduction in peripheral vascular 
TABLE 4: Changes in cardiovascular parameters, quality of life, and depression score.

\begin{tabular}{lcccc}
\hline \multirow{2}{*}{ Item } & \multicolumn{2}{c}{ Testosterone group } & \multicolumn{2}{c}{ Placebo group } \\
& Baseline & Endpoint & Baseline & Endpoint \\
\hline Quality of life & $128.96 \pm 4.91$ & $130.95 \pm 2.84$ & $128.00 \pm 4.56$ & $127.50 \pm 3.44$ \\
Beck score & $4.60 \pm 4.41$ & $5.00 \pm 6.28$ & $4.63 \pm 3.14$ & $5.55 \pm 5.50$ \\
End diastolic diameter & $5.94 \pm 1.06$ & $5.86 \pm 0.98$ & $5.90 \pm 0.82$ & $5.66 \pm 0.79$ \\
End systolic diameter & $4.92 \pm 1.02$ & $4.66 \pm 1.06$ & $6.87 \pm 1.27$ & $4.49 \pm 0.89$ \\
End diastolic volume & $141.44 \pm 54.06$ & $137.28 \pm 48.56$ & $149.16 \pm 46.51$ & $130.26 \pm 50.72$ \\
End systolic volume & $88.72 \pm 35.63$ & $87.76 \pm 40.80$ & $94.56 \pm 43.75$ & $87.04 \pm 36.40$ \\
Ejection fraction & $34.52 \pm 7.39$ & $37.12 \pm 8.23$ & $30.76 \pm 7.99$ & 0.440 \\
Tei index* & $0.54 \pm 0.02$ & $0.47 \pm 0.01$ & $0.55 \pm 0.02$ & 0.557 \\
\hline
\end{tabular}

${ }^{*}$ The pulsed Doppler Tei index is a parameter to evaluate combined systolic and diastolic cardiac performance that is measured by dividing Isovolumic contraction time plus isovolumic relaxation time by ejection time.

Data are presented as mean $\pm \mathrm{SD}$.

TABLE 5: Improvement in quality of life following testosterone therapy adjusted for baseline variables using the multivariate linear regression modeling.

\begin{tabular}{lcc}
\hline Item & Standardized beta & $P$ value \\
\hline Testosterone therapy & 0.419 & 0.035 \\
Advanced age & 0.319 & 0.195 \\
Body mass index & 0.015 & 0.936 \\
Diabetes mellitus & 0.022 & 0.912 \\
Hypertension & 0.236 & 0.296 \\
Hyperlipidemia & 0.124 & 0.514 \\
Cigarette smoking & 0.062 & 0.719 \\
NYHA classification & 0.070 & 0.706 \\
\hline
\end{tabular}

$R$-square $=0.436$.

* The beta coefficient is used to compare the strength of a predictor within the model to predict the dependant variable or outcome so that the higher beta indicates more power of the variable to predict outcome. Because beta coefficient is potentially affected by the standard error of the variable, the standardized beta adjusted for this error is considered.

${ }^{\dagger}$ A $P$ value lees than 0.05 indicates a significant role for predicting outcome by the considered variable.

resistance and increased coronary blood flow through vasodilation leading improvement of functional and symptomatic status [31]. Also, some effective role of this therapeutic regimen in preventing the occurrence of serious arrhythmia can be explainable by a direct regulative effect of testosterone on shorten Q-T intervals [32]. Moreover, association between the lack of testosterone and stimulating inflammatory responses has been shown and thus a remarkable improvement of cardiac performance and a significant decrease in the level of serum TNF-alpha following testosterone therapy has been suggested [33]. Along with the pointed mechanisms, a rise in hemoglobin [34], improvement of baroreceptor sensitivity [35], increase of muscle sympathetic nerve activity, and an increase of muscle arteriole vasodilation and function [36] have been also proposed. In addition, because insulin resistance is a major metabolic disturbance occurring in up to $40 \%$ of heart failure patients [37], this abnormality has been shown to be associated with low testosterone level leading decreased glucose utilization by skeletal muscle that results in muscle fatigue and wasting $[38,39]$. Thus, testosterone therapy can be potentially increase insulin sensitivity and thus improve glucose uptake by muscles and reduced disabilities. It seems that all beneficial cardiovascular, hormonal, and metabolic effects of testosterone injection can explain considerable improvement of quality of life in these patients.

According to the current understanding of testosterone replacement therapy with the goal of achieving testosterone levels that are consistently in the normal range pointed to in ISSAM recommendations [40], we applied low dose of testosterone in our trial. In some other experiments, testosterone enanthate was injected every 10 to 14 days [41]. Thus, we should apply higher dosages of testosterone enanthate with the aim of assessing real effects of this drug on study parameters. However, because of the lack of measuring baseline level of testosterone, nonexistence of monitoring of serum level of the drug, and lack of awareness of the drug effects in heart failure patients with an unusual clinical condition, we preferred to consider lower doses of this drug in our experiment. In addition, despite considering its low dose, useful effects of testosterone therapy on different physical and psychological aspects in heart failure patients were demonstrated.

As a potential limitation, we could not assess baseline testosterone level due to the high cost of these tests in our regions. It seems that the measurement of baseline serum level of testosterone could be useful to assess modification of the study clinical parameters along with monitoring changes in level of testosterone in serum. Furthermore, considering both hypogonadal and eugonadal subjects could result in providing a more comprehensive view of physiological effects of testosterone and other androgen endogenous hormones on cardiovascular parameters in CHF patients.

In conclusion, testosterone deficiency plays the major role in the pathophysiology of congestive heart failure, insights into the beneficial role of testosterone in improvement of function capacity, quality of life, and psychological state in these patients are strengthened. The present study attempted to demonstrate useful effects of testosterone therapy on different physical and psychological aspects in heart failure 
patients that result in confirming improvement of functional capacity and quality of life in these patients. Improvement in the Tei index consider as an indictor for early improvement in the cardiac function. Since Tei index shows very fine and subtle changes at the beginning of cardiac function so it needs to follow the patients for longer time.

\section{Conflict of Interests}

The authors declare that there is no conflict of interests regarding the publication of this paper.

\section{Acknowledgments}

This study was supported by the Islamic Azad University, Najafabad branch, Isfahan. The authors thank the university authorities that offered critical administrative support and managerial services for carrying out the study and also all researchers for their help and support. The authors also thank the Caspian Pharmaceutical Company for the supply of needed medications.

\section{References}

[1] V. L. Roger, S. A. Weston, M. M. Redfield et al., "Trends in heart failure incidence and survival in a community-based population," The Journal of the American Medical Association, vol. 292, no. 3, pp. 344-350, 2004.

[2] J. A. Ezekowitz, "A new pathway? Failure, fragility and fractures," European Heart Journal, vol. 31, no. 1, pp. 9-11, 2010.

[3] M. S. Nieminen, M. Böhm, M. R. Cowie et al., "Executive summary of the guidelines on the diagnosis and treatment of acute heart failure: the Task Force on Acute Heart Failure of the European Society of Cardiology," European Heart Journal, vol. 26, no. 4, pp. 384-416, 2005.

[4] C. J. Malkin, P. J. Pugh, J. N. West, E. J. R. van Beek, T. H. Jones, and K. S. Channer, "Testosterone therapy in men with moderate severity heart failure: a double-blind randomized placebo controlled trial," European Heart Journal, vol. 27, no. 1, pp. 57-64, 2006.

[5] E. A. Jankowska, B. Biel, J. Majda et al., "Anabolic deficiency in men with chronic heart failure: prevalence and detrimental impact on survival," Circulation, vol. 114, no. 17, pp. 1829-1837, 2006.

[6] B. Tappler and M. Katz, "Pituitary-gonadal dysfunction in lowoutput cardiac failure," Clinical Endocrinology, vol. 10, no. 3, pp. 219-226, 1979.

[7] E. A. Jankowska, G. Filippatos, B. Ponikowska et al., "Reduction in circulating testosterone relates to exercise capacity in men with chronic heart failure," Journal of Cardiac Failure, vol. 15, no. 5, pp. 442-450, 2009.

[8] C. J. Malkin, P. J. Pugh, P. D. Morris, S. Asif, T. H. Jones, and K. S. Channer, "Low serum testosterone and increased mortality in men with coronary heart disease," Heart, vol. 96, no. 22, pp. 1821-1825, 2010.

[9] C. J. Malkin, P. J. Pugh, P. D. Morris et al., “Testosterone replacement in hypogonadal men with angina improves ischaemic threshold and quality of life," Heart, vol. 90, no. 8, pp. 871-876, 2004.
[10] C. J. Malkin, P. J. Pugh, R. D. Jones, D. Kapoor, K. S. Channer, and T. H. Jones, "The effect of testosterone replacement on endogenous inflammatory cytokines and lipid profiles in hypogonadal men," Journal of Clinical Endocrinology and Metabolism, vol. 89, no. 7, pp. 3313-3318, 2004.

[11] P. A. McKee, W. P. Castelli, P. M. McNamara, and W. B. Kannel, "The natural history of congestive heart failure: the Framingham study," The New England Journal of Medicine, vol. 285, no. 26, pp. 1441-1446, 1971.

[12] F. Rashidi, A. Rashidi, A. Golmohamadi et al., "Does absolute neutrophilia predict early congestive heart failure after acute myocardial infarction? A cross-sectional study," Southern Medical Journal, vol. 101, no. 1, pp. 19-23, 2008.

[13] E. Barrett-Connor, E. V. Giardina, A. K. Gitt, U. Gudat, H. O. Steinberg, and D. Tschoepe, "Women and heart disease: the role of diabetes and hyperglycemia," Archives of Internal Medicine, vol. 164, no. 9, pp. 934-942, 2004.

[14] D. Wood, G. de Backer, O. Faergeman, I. Graham, G. Mancia, and K. Pyörälä, "Prevention of coronary heart disease in clinical practice. Recommendations of the Second Joint Task Force of European and other Societies on Coronary Prevention," European Heart Journal, vol. 19, no. 10, pp. 1434-1503, 1998.

[15] M. Bartnik, L. Rydén, R. Ferrari et al., "The prevalence of abnormal glucose regulation in patients with coronary artery disease across Europe: The Euro Heart Survey on diabetes and the heart," European Heart Journal, vol. 25, no. 21, pp. 1880-1890, 2004.

[16] J. Chalmers, S. MacMahon, G. Mancia et al., "World Health Organization-International Society of Hypertension Guidelines for the management of hypertension. Guidelines subcommittee of the World Health Organization," Clinical and Experimental Hypertension, vol. 21, no. 5-6, pp. 1009-1060, 1999.

[17] American Diabetes Association, "Diagnosis and Classification of Diabetes Mellitus,” Diabetes Care, vol. 31, pp. 55-60, 2008.

[18] B. Pollentier, S. L. Irons, C. M. Benedetto et al., "Examination of the six minute walk test to determine functional capacity in people with chronic heart failure: a systematic review," Cardiopulmonary Physical Therapy Journal, vol. 21, no. 1, pp. 13-21, 2010.

[19] M. H. Picard, D. Adams, S. M. Bierig et al., "American society of echocardiography recommendations for quality echocardiography laboratory operations," Journal of the American Society of Echocardiography, vol. 24, no. 1, pp. 1-10, 2011.

[20] M. Gharipour, R. Kelishadi, A. Khosravi, S. Shirani, M. Masjedi, and N. Sarrafzadegan, "The impact of a community trial on the pharmacological treatment in the individuals with the metabolic syndrome: findings from the Isfahan Healthy Heart Program, 2001-2007," Archives of Medical Science, vol. 8, no. 6, pp. 10091017, 2012.

[21] H. HosseinKaviani and A. S. Mousavi, "Psychometric properties of the Persian version of Beck Anxiety Inventory (BAI)," Tehran University Medical Journal, vol. 65, pp. 136-140, 2008.

[22] A. T. Beck, C. H. Ward, M. Mendelson, J. Mock, and J. Erbaugh, "An inventory for measuring depression," Archives of General Psychiatry, vol. 4, pp. 561-571, 1961.

[23] N. N. Hairi, R. G. Cumming, V. Naganathan et al., "Loss of muscle strength, mass (sarcopenia), and quality (specific force) and its relationship with functional limitation and physical disability: the concord health and ageing in men project," Journal of the American Geriatrics Society, vol. 58, no. 11, pp. 2055-2062, 2010. 
[24] A. Rydlewska, J. Maj, B. Katkowski et al., "Circulating testosterone and estradiol, autonomic balance and baroreflex sensitivity in middle-aged and elderly men with heart failure," Aging Male, vol. 16, no. 2, pp. 58-66, 2013.

[25] C. T. Nguyen, A. Aaronson, R. P. Morrissey, M. Agarwal, R. D. Willix, and E. R. Schwarz, "Myths and truths of growth hormone and testosterone therapy in heart failure," Expert Review of Cardiovascular Therapy, vol. 9, no. 6, pp. 711-720, 2011.

[26] A. J. Aaronson, R. P. Morrissey, C. T. Nguyen, R. Willix, and E. R. Schwarz, "Update on the safety of testosterone therapy in cardiac disease," Expert Opinion on Drug Safety, vol. 10, no. 5, pp. 697-704, 2011.

[27] M. Stout, G. A. Tew, H. Doll et al., “Testosterone therapy during exercise rehabilitation in male patients with chronic heart failure who have low testosterone status: a double-blind randomized controlled feasibility study," American Heart Journal, vol. 164, no. 6, pp. 893-901, 2012.

[28] F. Iellamo, M. Volterrani, G. Caminiti et al., "Testosterone therapy in women with chronic heart failure: a pilot doubleblind, randomized, placebo-controlled study," The Journal of the American College of Cardiology, vol. 56, no. 16, pp. 1310-1316, 2010.

[29] M. Toma, F. A. McAlister, E. E. Coglianese et al., "Testosterone supplementation in heart failure a meta-analysis," Circulation: Heart Failure, vol. 5, no. 3, pp. 315-321, 2012.

[30] E. A. Jankowska, G. Filippatos, B. Ponikowska et al., "Reduction in circulating testosterone relates to exercise capacity in men with chronic heart failure," Journal of Cardiac Failure, vol. 15, no. 5, pp. 442-450, 2009.

[31] C. T. Nguyen, A. Aaronson, R. P. Morrissey, M. Agarwal, R. D. Willix, and E. R. Schwarz, "Myths and truths of growth hormone and testosterone therapy in heart failure," Expert Review of Cardiovascular Therapy, vol. 9, no. 6, pp. 711-720, 2011.

[32] J. B. Schwartz, M. Volterrani, G. Caminiti et al., "Effects of testosterone on the Q-T interval in older men and older women with chronic heart failure," International Journal of Andrology, vol. 34, no. 5, pp. e415-e421, 2011.

[33] Z. B. Li, J. Wang, J. X. Wang, X. M. Chen, and S. S. Jiang, "Testosterone therapy improves cardiac function of male rats with right heart failure," Zhonghua nan ke xue, vol. 15, no. 11, pp. 994-1000, 2009.

[34] M. H. Emmelot-Vonk, H. J. Verhaar, H. R. Nakhai Pour et al., "Effect of testosterone supplementation on functional mobility, cognition, and other parameters in older men: a randomized controlled trial," The Journal of the American Medical Association, vol. 299, no. 1, pp. 39-52, 2008.

[35] F. Iellamo, M. Volterrani, G. Caminiti et al., "Testosterone therapy in women with chronic heart failure: a pilot doubleblind, randomized, placebo-controlled study," The Journal of the American College of Cardiology, vol. 56, no. 16, pp. 1310-1316, 2010.

[36] G. Caminiti, M. Volterrani, F. Iellamo et al., "Effect of longacting testosterone treatment on functionalexercise capacity, skeletal muscle performance, insulin resistance, and baroreflexsensitivity in elderly patients with chronic heart failure a double-blind, placebocontrolled,randomized study," The Journal of the American College of Cardiology, vol. 54, no. 10, pp. 919-927, 2009.

[37] N. Suskin, R. S. McKelvie, R. J. Burns et al., "Glucose and insulin abnormalities relate to functional capacity in patients with congestive heart failure," European Heart Journal, vol. 21, no. 16, pp. 1368-1375, 2000.
[38] W. Doehner, D. Gathercole, M. Cicoira et al., "Reduced glucose transporter GLUT4 in skeletal muscle predicts insulin resistance in non-diabetic chronic heart failure patients independently of body composition," International Journal of Cardiology, vol. 138, no. 1, pp. 19-24, 2010.

[39] C. J. Malkin, T. H. Jones, and K. S. Channer, “Testosterone in chronic heart failure," Frontiers of Hormone Research, vol. 37, pp. 183-196, 2009.

[40] C. Wang, E. Nieschlag, R. S. Swerdloff et al., "ISA, ISSAM, EAU, EAA and ASA recommendations: investigation, treatment and monitoring of late-onset hypogonadism in males," Aging Male, vol. 12, no. 1, pp. 5-12, 2009.

[41] E. Nieschlag, H. J. Cüeppers, W. Wiegelmann, and E. J. Wickings, "Bioavailability and LH suppressing effect of different testosterone preparations in normal and hypogonadal men," Hormone Research, vol. 7, no. 3, pp. 138-145, 1976. 


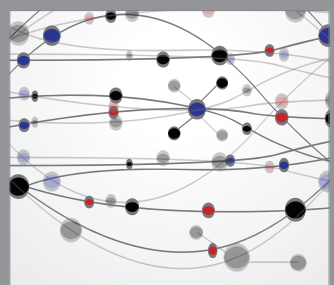

The Scientific World Journal
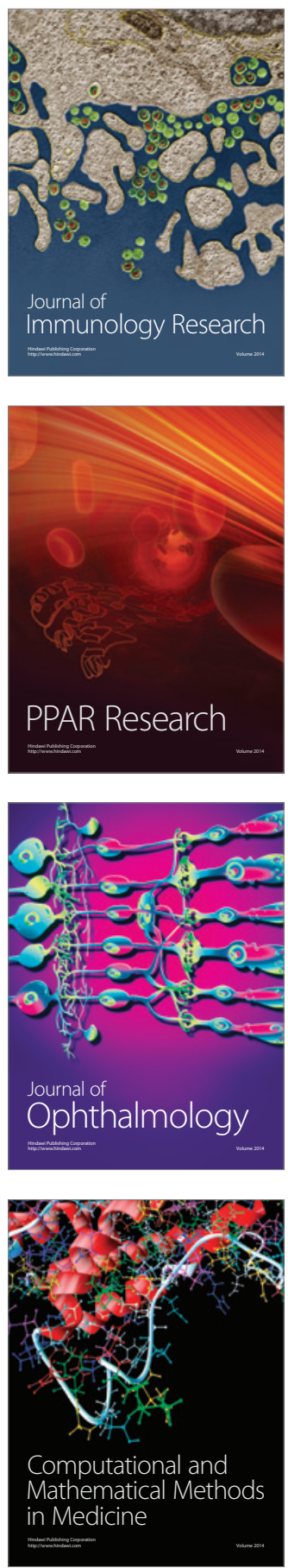

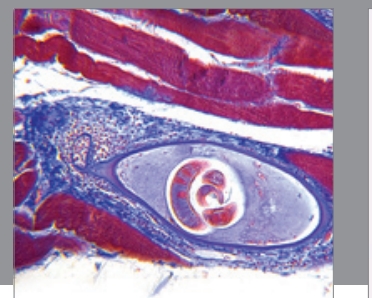

Gastroenterology

Research and Practice
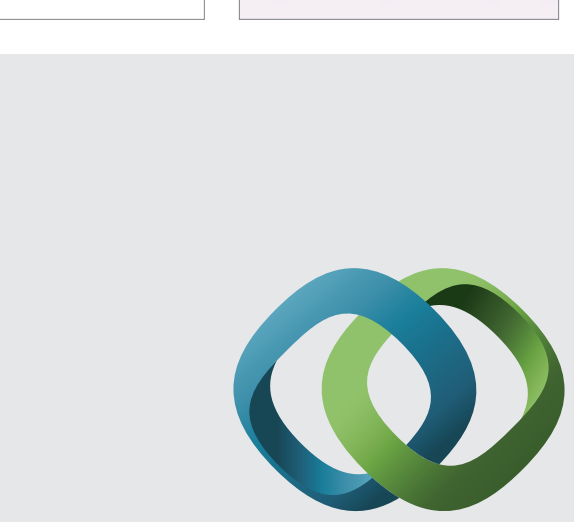

\section{Hindawi}

Submit your manuscripts at

http://www.hindawi.com
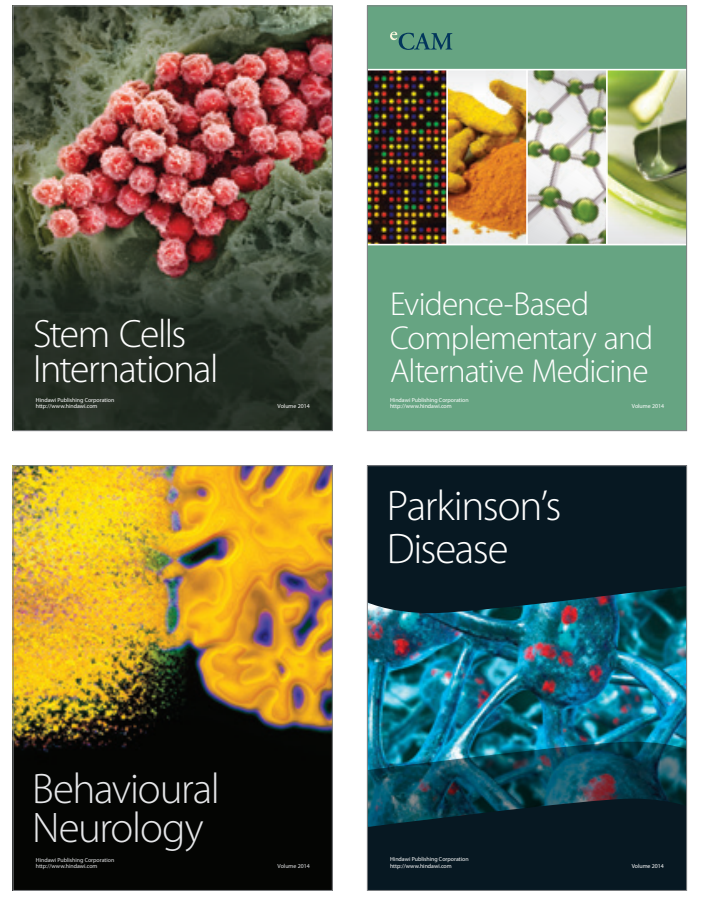
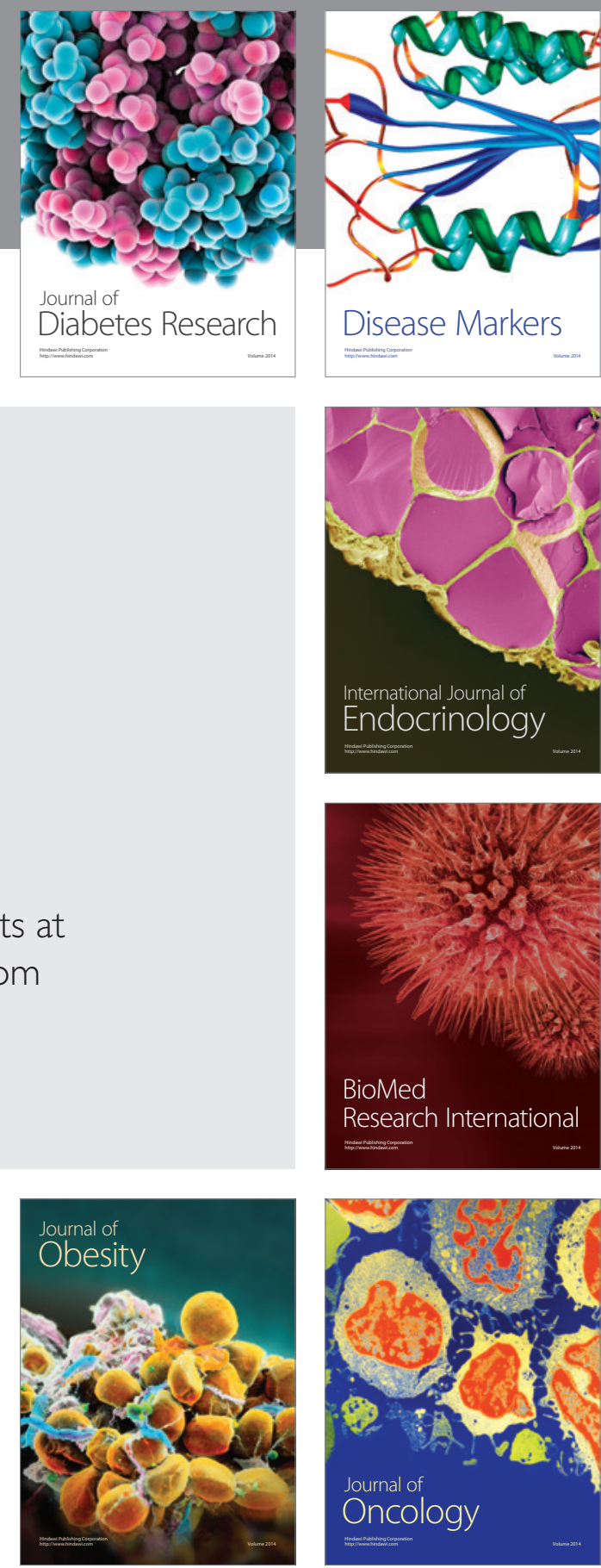

Disease Markers
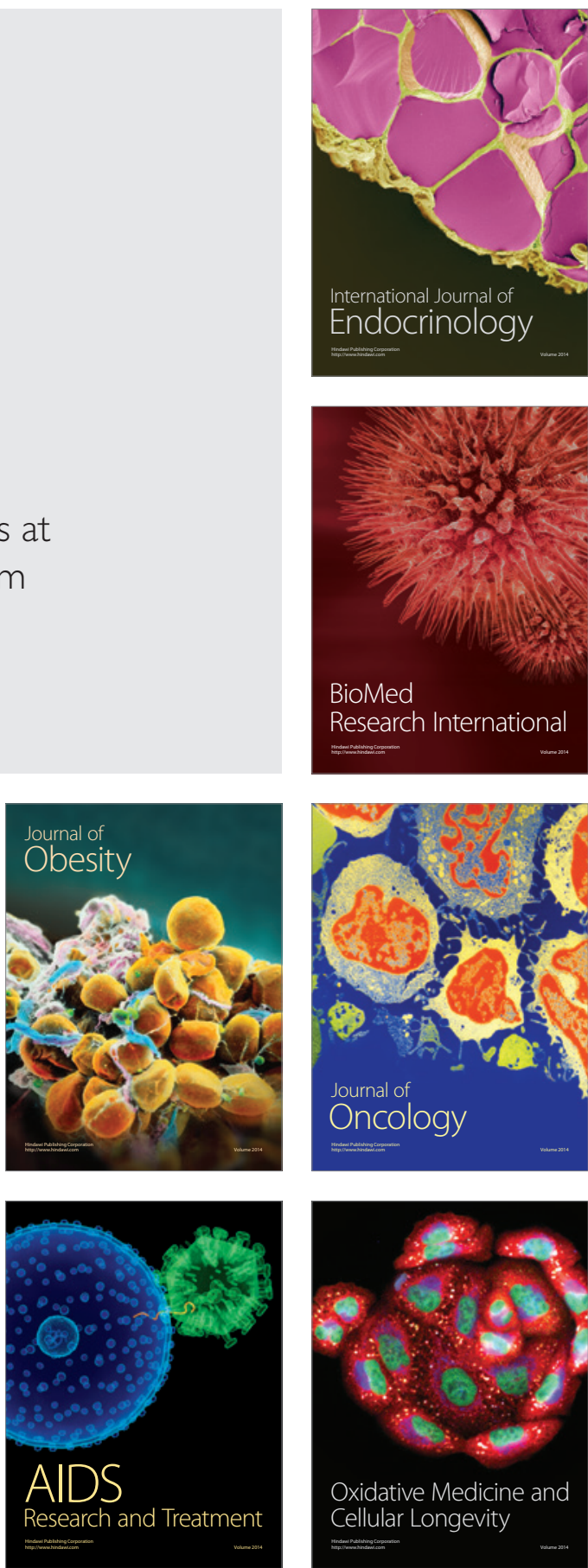